causo as that which I have studied at Amani. Doubt. less several forms of leaf-curling occur, attributable to different causes. Workers in South Africa have recognised two types; one, 'curly-leaf', shown by them to be an hereditary abnormality, and a socond, 'crinkly-dwarf ', of unknown cause. ${ }^{1}$ Their description of 'crinkly-dwarf' agrees well with the Amani disease. Roberts ${ }^{2}$ attributed a leaf-curl of tobacco in Southern Rhodesia to injury by a capsid bug. Other workers have regarded leaf-curling as a reaction to unfavourable soil conditions. It is possible that the investigation of some of the curly-leaf conditions elsewhere upon the lines which I have followed may demonstrate their identity with the Amani disease.

It is a matter of interest that another whiteflytransmitted virus disease, the leaf-crinkle of cotton, is also characterised by leafy outgrowths from the lower surface of the leaves. ${ }^{3}$

East African Agricultural

Research Station,

Amani, Tanganyika Territory, June 17.

1 Rept. of Proceedings, Pan-African Agricultural and Veterinary Conference, Pretoria, 1929, p. 91.

p1 169.183

- Kirkpatrick, Bull. Ent. Res., 21, pp. 127-137.

\section{Intensity Anomalies in Rare Gas Spectra.}

Is Paschen's analysis of the neon spectrum, and in Meissner's analysis of the argon spectrum, there occur curious irregularities in the $d$-term sequences, and similar deviations are shown in the spectra of krypton and xenon investigated by Meggers, de Bruin, and Humphreys.

An extension of the system of $d$-terms in rare gas spectra, to be published elsewhere by one of us (E. R.), has shown intensity irregularities running parallel with the deviations in the term values. The argon spectrum will serve as an example.

The two terms 3302.90 and 1828.40 , the classifica. tion of which has hitherto been uncertain, are now, due to the observation of several new combinations, definitely classified as $6 d_{3}$ and $8 d_{3}$. These two terms deviate strongly from the Ritz-formula. Comparison was then made of the intensities of the lines in the series $2 p_{10}-m d_{5}$ and in $2 p_{10}-m d_{3}$, which for $m=5 \cdot 7$ and 9 are about equally strong, but for $m=6$ and 8 the combinations are found to be much weaker with $d_{s}$ than with $d_{s}$. That is to say, intensity anomalies take place for those terms where the greatest deviations from Ritz's formula occur. Exactly the same thing is found in the spectrum of krypton.

In a theoretical interpretation of the origin of these term anomalies a study of the intensity anomalies is certain to play an important part. Therefore one of us (H. S.), engaged in photographic intensity measurements in rare gas spectra, has undertaken quantitative measurements also for the mentioned combinations.

The following intensity ratios indicate the type of deviations which are found :

$$
\frac{I\left(2 p_{10}-5 d_{3}\right)}{I\left(2 p_{10}-5 d_{5}\right)}=\frac{1}{1} \quad \frac{I\left(2 p_{10}-6 d_{3}\right)}{I\left(2 p_{10}-6 d_{5}\right)}=\frac{1}{6} \quad \frac{I\left(2 p_{10}-7 d_{3}\right)}{I\left(2 p_{10}-7 d_{5}\right)}=\frac{1}{1}
$$

Further measurements are in progress.

We are indebted to Prof. Bohr for his interest in this problem, and one of us (H.S.) has had the privilege of $\&$ fellowship from the American-Scandinavian Foundation.

University Institute for Theoretical EBBE RASMUSSEN. Hugo SwENSON. Physics, Copenhagen, June 25.
Effect of Pressure on Raman Spectra.

THF influonce of its neighbours on the rotation and vibration states of a molecule in a gas is due partly to the general intermolecular field and partly to actual collisions. From the kinetic theory of gases, it is known that the mean frequency of collisions which a molecule undergoes is given by the ratio $P / \eta$ of the pressure to the viscosity of the gas. The perturbing influence of collisions on molecular rotation may be expectod to bo small so long as this frequency is low, but would become considerable when the mean frequency of collision approaches a value comparable to that of molecular rotation. By taking $P / \eta$ to be equal to the frequency of rotation of the molecule in the one-quantum state, we may obtain an estimate of the pressure at which the distinctness of quantisation of the different rotations would disappear. In this way, the following estimates of pressure in atmospheres at $30^{\circ} \mathrm{C}$. are obtained for the different gases: Hydrogen, 450; methane, 48; nitrogen, 29 ; oxygen, 25 ; acetylene, 10 ; carbon dioxide, 5 ; hydrogen chloride, 128 ; ammonia, 83 ; nitric oxide, 25.

These estimates agree remarkably well with actual observations of the pressures above which the discrete lines in the rotational Raman spectrum of the gas disappear and are replaced by a continuous spectrum. I have found that oxygen and nitrogen show the rotational fine structure at a pressure of 20 atm., while only an unresolved band is obtained at a pressure of $30 \mathrm{~atm}$. Houston and Lewis report a similar result for carbon dioxide above 5 atm. pressure. I In the other cases, the calculated pressures are uniformly greater than those at which Rasetti and others have actually succeeded in recording the rotational fine structure. It is not improbable that in the case of polar molecules the fine structure might disappear at somewhat lower pressures than those given above, in consequence of the intermolecular fields.

\section{Bowbazar Street,} Calcutta, India, June 22.

I Proc. Nat. Acad., vol, 17, p. 229 ; 1931.

\section{The Slow Combustion of Methane and Ethane.}

NOT knowing on what experimental evidence (if any) Dr. Mardles 1 bases his conclusions about the initial 'peroxidation' of methane and ethane to 'methyl hydrogen peroxide' and 'ethyl hydrogen peroxide' respectively during slow combustion in air, may I inquire whether (and, if so, how) he has isolated and identified any such substances so produced? For, as was pointed out in the paper on "The Slow Combustion of Ethane" recently pub. lished by Mr. S. G. Hill and me, ${ }^{2}$ there has been, so far, no real experimental proof about the initial 'peroxidation' of the hydrocarbons in question in slow combustion; although, as was then shown, the fact that in the case of ethane some slight "peroxida. tion' of the intermedially formed acetaldehyde may occur has perhaps confused the issue in some minds and misled some observers.

All I can testify is that, as the outcome of many years' careful experimental study of the combustion of methane and ethane over a very wide range of conditions in my laboratories (both earlier on in Manchester and more recently here in London), no evidence whatever of an initial 'peroxidation' has yet been discovered; on the contrary, the weight of cumulative evidence is so overwhelmingly in favour

No. 3222, VoL. 128] 